\title{
Pharmacokinetics, Safety, and Preliminary Efficacy of Oral Trifluridine/Tipiracil in Chinese Patients with Solid Tumors: A Phase Ib, Open-Label Study
}

This article was published in the following Dove Press journal: Clinical Pharmacology: Advances and Applications

\author{
Xicheng Wang $\mathbb{D}^{\prime}$ \\ Jianfeng Zhou $\mathbb{D D}^{2}$ \\ Yan $\mathrm{Li}^{1}$ \\ Yuping $\mathrm{Ge}^{2}$ \\ Yanping Zhou ${ }^{2}$ \\ Chunmei Bai (iD) ${ }^{2}$ \\ Lin Shen (ID)
}

'Key Laboratory of Carcinogenesis and Translational Research (Ministry of Education), Department of Gastrointestinal Oncology, Peking University Cancer Hospital \& Institute, Beijing 100|42, People's Republic of China; ${ }^{2}$ Department of Oncology, Peking Union Medical College Hospital, Beijing 100032, People's Republic of China

Correspondence: Lin Shen

Key Laboratory of Carcinogenesis and

Translational Research (Ministry of

Education), Department of

Gastrointestinal Oncology, Peking

University Cancer Hospital \& Institute,

No. 52, Fu Cheng Road, Hai Dian District,

Beijing, People's Republic of China

Tel +86-010-88156175

Fax +86-010-88|9656।

Email linshenpku@I63.com

Chunmei Bai

Department of Oncology, Peking Union

Medical College Hospital, No. 4I, Da Mu

Cang Hutong, Xi Cheng District, Beijing,

People's Republic of China

$\mathrm{Tel} / \mathrm{Fax}+86-010-69158315$

Email baichunmeil964@I63.com
Purpose: Trifluridine/tipiracil (FTD/TPI) is approved in Japan, the United States (US), and Europe for metastatic colorectal cancer (mCRC) refractory to standard therapies. This Phase $1 \mathrm{~b}$ open-label study focused on the pharmacokinetic (PK) and toxicity profiles of FTD/TPI in Chinese patients with solid tumors.

Methods: Patients with definitive histologically or cytologically confirmed advanced/metastatic solid tumors refractory to standard treatments were enrolled. FTD/TPI $\left(35 \mathrm{mg} / \mathrm{m}^{2}\right)$ was administered orally twice daily for five consecutive days, followed by a 2-day recovery. Treatment was repeated for five consecutive days, followed by a 16-day recovery. The primary objective was to assess PK characteristics of FTD, 5-trifluoromethyl-2,4 $(1 \mathrm{H}, 3 \mathrm{H})$ pyrimidinedione (FTY; an inactive form of FTD), and TPI, calculated from plasma concentrations. Additionally, these PK values were compared with those from similar Phase 1 studies in patients from Japan and the US, using Tukey-Kramer's honestly significant difference (HSD) multiple comparison tests. Safety and preliminary efficacy of FTD/TPI were assessed.

Results: Fifteen patients (12 males, three females) were enrolled, most with CRC (87\%). Geometric mean analysis showed that maximum plasma concentration $\left(\mathrm{C}_{\max }\right)$ of FTD increased after multiple administration (from day $1[3019.5 \mathrm{ng} / \mathrm{mL}]$ to day $12[3693.1 \mathrm{ng} / \mathrm{mL}]$ ), and the exposure $\left(\mathrm{AUC}_{0-\mathrm{t}}\right)$ increased 2.4-fold (day 1:7796.6 ng/mL•h; day 12:18,181.3 ng/mL•h). There was no meaningful change in the exposure to FTY and TPI throughout the study. HSD tests showed comparable PK for FTD, FTY, and TPI between Chinese and Japanese patients, and comparable exposure to FTD between Chinese and US patients. Eight patients (53.3\%) experienced Grade 3 treatment-emergent adverse events, most frequently anemia and fatigue $(13.3 \%$, two events each). Median progression-free survival was 1.9 months.

Conclusion: FTD/TPI had an acceptable safety and efficacy profile and PK characteristics were comparable between Chinese, Japanese, and US patients, suggesting that this treatment may be suitable for Chinese patients with refractory mCRC.

Trial Registration: This trial was registered at clinicaltrials.gov as NCT02261532.

Keywords: Chinese, colorectal cancer, pharmacokinetics, safety, trifluridine/tipiracil

\section{Introduction}

Colorectal cancer (CRC) is the third most common cancer and the second leading cause of cancer-related mortality worldwide. ${ }^{1}$ Based on the most recent GLOBOCAN global estimates, there were over 1.8 million new cases of CRC (representing approximately $10 \%$ of all new cancers), and over 861,000 deaths in 2018. ${ }^{1}$ Early-stage CRC and advanced resectable disease can usually be managed through surgery with preoperative, perioperative, or postoperative treatment 
including chemotherapy with or without radiation therapy, depending on regional guidelines and tumor stage. ${ }^{2-5}$ For metastatic CRC (mCRC), the backbone of the current firstline standard of care is 5-fluorouracil (5-FU) and folinic acid with the addition of either irinotecan or oxaliplatin and/or without vascular endothelial growth factor inhibitor. If the tumor contains a wild-type RAS gene, an epidermal growth factor inhibitor is added to the treatment. ${ }^{2-5}$ Among newer subsequent therapy options for mCRC, trifluridine/tipiracil (FTD/TPI) has become available in the United States (US), Europe, and Japan for patients whose disease progressed following standard therapies. ${ }^{2-5}$

FTD/TPI, also known as TAS-102, is an oral formulation composed of FTD, a thymidine-based, antineoplastic nucleoside analog, and TPI, a thymidine phosphorylase (TPase) inhibitor. The mechanism of cytotoxic action of FTD is different from that of 5-FU, ${ }^{6,7}$ and is based on creating DNA dysfunction through the incorporation of the triphosphate form of FTD, following phosphorylation by thymidine kinase-1. ${ }^{6,8,9}$ FTD incorporated in DNA was correlated with antitumor effects in vivo using human cancer xenografts in nude mice. ${ }^{8}$ FTD is also known to induce transient phosphorylation of Chk1, accumulation of $\mathrm{p} 53$ and $\mathrm{p} 21$ proteins in p53-proficient human cancer cell lines, leading to a sustained G2 phase arrest. ${ }^{10}$ However, FTD is rapidly degraded by hepatic and intestinal TPase into an inactive 5-trifluoromethyl-2,4 (1H,3H)-pyrimidinedione (FTY), which results in low concentration of FTD., ${ }^{6,11,12}$ Combining FTD with TPI at a 1:0.5 molar ratio inhibited degradation of FTD and potentiated antitumor activity of FTD. ${ }^{12,13}$ In preclinical studies, FTD/TPI was active in fluorouracil-resistant colorectal cancer cell lines, with a growth inhibition pattern different from that of 5-FU, ${ }^{7}$ and in human gastric and colorectal 5-FU-resistant tumors xenografted into nude mice. ${ }^{13}$ These findings established FTD/TPI combination as an attractive candidate for the treatment of patients with $\mathrm{mCRC}$ refractory to fluoropyrimidines.

Phase 1 clinical studies evaluated FTD/TPI pharmacokinetic (PK) characteristics, dose-limiting toxicities (DLTs) and recommended dosage of FTD/TPI in patients with advanced solid tumors and mCRC. ${ }^{14-19}$ Exposure to FTD increased with increased doses and repeated administration; ${ }^{14,16-18}$ and, based on the DLTs, the $35 \mathrm{mg} / \mathrm{m}^{2}$ twice-daily dosage was selected for the Phase 2/3 studies. ${ }^{18,19}$ A proof-of-concept Phase 1 study in patients with solid tumors conclusively demonstrated that administration of FTD+TPI at $35 \mathrm{mg} / \mathrm{m}^{2}$ twice daily increased exposure to FTD by 37 -fold, compared with administration of FTD alone. ${ }^{17}$ The clinical efficacy of
FTD/TPI was not affected by food intake. ${ }^{20}$ Major DLTs associated with FTD/TPI treatment were mainly hematologic and included granulocytopenia, neutropenia, anemia, leucopenia, thrombocytopenia, lymphopenia, and febrile neutropenia. ${ }^{15,16,18,19}$

FTD/TPI at a $35 \mathrm{mg} / \mathrm{m}^{2}$ twice-daily dosage demonstrated substantial antitumor activity in several randomized clinical trials in Western and Asian patients with refractory mCRC. FTD/TPI demonstrated significant improvement in overall survival (OS) vs placebo in heavily pretreated Japanese patients with mCRC refractory to 5-FU, irinotecan, and oxaliplatin (hazard ratio [HR], 0.56; 95\% confidence interval [CI], $0.39-0.81 ; p=0.001) ;{ }^{21}$ in patients with refractory $\mathrm{mCRC}$ from Australia, Europe, Japan, and the US (RECOURSE global trial: HR, $0.68 ; 95 \%$ CI, $0.58-0.81 ; p<0.001$; and RECOURSE Spanish subpopulation: HR, 0.47; 95\% CI, 0.$28-0.78 ; p=0.003) ; 22,23$ and in Asian patients from China, Republic of Korea, and Thailand, with previously treated mCRC (TERRA trial: HR, 0.79; 95\% CI, 0.62-0.99; $p=0.035){ }^{24,25}$ Among 305 Chinese patients enrolled in the TERRA study (75\% of all patients), 204 were treated with FTD/TPI and 101 received placebo. Compared to the placebo group, FTD/TPI reduced the risk of death by $18 \%$ (HR, 0.82 ; 95\% CI, 0.62-1.08) in Chinese patients. ${ }^{25}$ FTD/TPI is currently approved in Japan, the US, and Europe for the treatment of patients with mCRC refractory to standard therapies, ${ }^{2-4}$ but this treatment is not yet approved in China.

We evaluated the PK profile, safety, and preliminary efficacy of FTD/TPI in Chinese patients with advanced/ metastatic solid tumors (predominantly CRC) refractory to standard treatments, as mandated by Chinese Food and Drug Administration (FDA) regulatory requirements to obtain approval for FTD/TPI treatment in China. To evaluate statistically whether the PK profile of FTD/TPI at a $35 \mathrm{mg} / \mathrm{m}^{2}$ twice-daily oral dosage in Chinese patients is comparable to that reported for Japanese and US patients in Phase I studies, ${ }^{17,18}$ we used the original data available for subsets of patients who were treated at this dose level (clinical study reports from JapicCTI-111545 and NCT01867866) and performed Tukey-Kramer's honestly significant difference (HSD) multiple comparison tests.

\section{Methods}

\section{Patients}

Patients eligible for enrollment to this study were 18-65 years of age, with histologically or cytologically confirmed advanced or metastatic solid tumors, who had either poor 
response to standard treatment, were intolerant to the currently available standard treatments, or had advanced or metastatic solid tumors for which no standard therapy was available. Additional inclusion criteria included a life expectancy of $\geq 3$ months, the ability to take medications orally, measurable and/or nonmeasurable lesions as defined by Response Evaluation Criteria In Solid Tumors (RECIST) v1.1 criteria, ${ }^{26}$ adequate organ function, and Eastern Cooperative Oncology Group performance status (ECOG PS) of 0 or 1.

Patients were excluded from the study if they had suffered serious complications that required hospitalization, had brain metastases or clinical symptoms suggestive of brain metastases, active infection, or a history of drug allergy, had undergone continuous systemic steroid therapy, or had a Grade $\geq 2$ unresolved toxicity per National Cancer Institute Common Terminology Criteria for Adverse Events $\left(\mathrm{CTCAEv} 4.03^{27}\right)$ attributed to any prior therapies.

\section{Study Design and Treatments}

This was an open-label, single-group, Phase 1b study of FTD/TPI in Chinese patients with solid tumors (NCT02261532). FTD/TPI (35 mg/m²) was administered orally twice daily (morning and evening) for five consecutive days, followed by 2 recovery days. The treatment was administered again for five consecutive days, followed by 16 days' recovery. This 28-day period was considered one treatment cycle. Study continued until all patients discontinued treatment, or after the last patient enrolled had received 12 months' study treatment, whichever occurred first.

The study protocol was approved by the Independent Ethics Committees in Beijing Cancer Hospital and Peking Union Medical College Hospital. The study was conducted in accordance with the provisions of the Declaration of Helsinki, the International Council for Harmonisation E6 Good Clinical Practice guidelines, and local regulatory requirements. ${ }^{28,29}$ Written informed consent was obtained from all patients before entering the study.

\section{Study Endpoints and Assessments}

The primary objective of this study was to investigate the PK characteristics of FTD, FTY, and TPI after single and multiple doses of FTD/TPI in Chinese patients. The following PK parameters of FTD, FTY, and TPI were calculated: maximum plasma concentration $\left(\mathrm{C}_{\max }\right)$, time to maximum concentration $\left(\mathrm{T}_{\max }\right)$, area under the plasma concentrationtime curve (AUC), AUC from time 0 to time of last quantifiable concentration $\left(\mathrm{AUC}_{0-\mathrm{t}}\right), \mathrm{AUC}$ from time 0 to time infinity $\left(\mathrm{AUC}_{0 \text {-inf }}\right)$, apparent volume of distribution volume $(\mathrm{Vd} / \mathrm{F})$, apparent systemic clearance $(\mathrm{CL} / \mathrm{F})$, terminal halflife $\left(\mathrm{t}_{1} / 2\right)$, elimination rate constant, and the ratio of $\mathrm{AUC}_{0-\mathrm{t}}$ on day 12 to $\mathrm{AUC}_{0-\mathrm{t}}$ on day $1(\mathrm{R})$. For FTY, neither $\mathrm{Vd} / \mathrm{F}$ nor $\mathrm{CL} / \mathrm{F}$ was calculated, and $\mathrm{AUC}_{0 \text {-inf was calculated only }}$ for day 1 .

Blood samples ( $\geq 4 \mathrm{~mL}$ ) for PK analyses were collected in the morning of days 1 and 12 of Cycle 1 at the following time points: 0,15 , and 30 mins, $1,2,4,6,8$, and 10 $\mathrm{h}$ after study drug administration. The sample at 0 mins was collected within 30 mins before the morning dose, and the sample at $10 \mathrm{~h}$ was collected before administration of the evening dose. The total blood volume collected for the PK samples was $72 \mathrm{~mL}$ per patient, with a minimum of $4 \mathrm{~mL}$ per sample.

The methods of liquid-liquid extraction for FTD and FTY and solid-phase extraction for TPI were applied, and the organic layer and eluate were collected and evaporated to dryness. These reconstituted samples were used for the analysis. FTD, FTY, and TPI concentrations were measured at the Toray Research Center (Kanagawa, Japan) using liquid chromatography with tandem mass spectrometry (LC/MS/MS) methods validated by the laboratory. FTD and FTY were extracted from the plasma samples with tertbutyl methyl ether and quantified by LC/MS/MS using internal standards labelled with stable isotopes. Lower limits of quantification (LLOQ) of FTD and FTY were $5.0 \mathrm{ng} /$ $\mathrm{mL}$. TPI was extracted from plasma samples using solid extraction columns and quantified by LC/MS/MS using an isotope-labelled internal standard. LLOQ of TPI was $0.2 \mathrm{ng} /$ $\mathrm{mL}$. For FTD, the intra-assay accuracy and precision at 5.0 $\mathrm{ng} / \mathrm{mL}$ were $103.0 \%$ and $6.2 \%$, respectively, and the interassay accuracy and precision rates were $101.2 \%$ and $6.7 \%$, respectively. For FTY, the intra-assay accuracy and precision rates at $5.0 \mathrm{ng} / \mathrm{mL}$ were $105.4 \%$ and $12.7 \%$, respectively, and the inter-assay accuracy and precision rates were $104.6 \%$ and $10.7 \%$, respectively. The intra-assay and interassay accuracy and precision rates for TPI at $0.2 \mathrm{ng} / \mathrm{mL}$ were $97.0 \%$ and $13.4 \%$ and $101.5 \%$ and $9.6 \%$, respectively. All data met the acceptance criteria.

Secondary endpoints included safety and preliminary efficacy of FTD/TPI in Chinese patients. For safety, adverse events (AEs), clinical laboratory tests, electrocardiograms, vital signs, and ECOG PS were assessed. All AEs, serious AEs, and deaths were recorded throughout the study and the follow-up period of 30 days after the last dose of study drug, or until the start of new antitumor therapy. At the end of the follow-up period, only AEs with 
a causal relationship to the study treatment were recorded. Adverse events were coded using Medical Dictionary for Regulatory Activities central coding dictionary version 18.0, and grading and severity of AEs were assessed using CTCAE v4.03. ${ }^{27}$

Efficacy endpoints included overall response rate (ORR), disease control rate (DCR), and progression-free survival (PFS). Tumor measurements were obtained using a contrast-enhanced computed tomography (CT) scan of the chest, abdomen, and pelvis within 28 days prior to enrollment and every 8 weeks $(+/-7$ days) thereafter. For patients who discontinued treatment for reasons other than radiologic disease progression, tumor measurements were continued every 8 weeks during the follow-up period until radiologic disease progression was documented. Tumor assessments were based on the individual investigators' review of the images according to RECIST v1.1.

\section{Statistical Analyses}

Fifteen evaluable patients were to receive the study drug. The overall sample size was not based on any formal hypothesis testing, but determined according to Chinese regulatory authority requirements, ${ }^{30,31}$ and aimed to obtain preliminary information on the PK and toxicity profile of FTD/TPI.

The PK population comprised all patients who received study medication. All such patients were evaluated for PK unless significant protocol deviations affected the data, or if key dosing information was missing. PK parameters were calculated from plasma concentrations using noncompartmental model analysis with commercial software (Phoenix WinNonlin v6.3, Pharsight Corporation as part of Certara, St Louis, MO, USA). $\mathrm{AUC}_{\mathrm{inf}}$ values were calculated for day 1 and day 12 using the following equation: $\mathrm{AUC}_{\mathrm{inf}}=\mathrm{AUC}_{0-\mathrm{t}}+$ $\mathrm{Ct} / \lambda \mathrm{z}$, where $\mathrm{Ct}$ and $\lambda \mathrm{z}$ mean the last quantifiable concentration and elimination rate constant, respectively. Among PK parameters for FTD, FTY, and TPI, $\mathrm{T}_{\max }$ was represented as mean or median, and $\mathrm{R}$ (ratio of $\mathrm{AUC}_{0-\mathrm{t}}$ on day 12 to $\mathrm{AUC}_{0-\mathrm{t}}$ on day 1), as well as other PK parameters, was described by the geometric mean values.

The safety population included all patients who received $\geq 1$ dose of study medication and the safety data were summarized descriptively. Compliance rate was based on drug accountability, calculated as the number of study drug doses taken (total dispensed $=$ [total returned + total lost]) divided by the prescribed number of doses expressed as a percentage.

The efficacy population included all patients in the safety population who had completed $\geq 1$ cycle of study medication and had assessments to confirm radiologic or clinical disease progression. Overall response rate (proportion of patients with complete response [CR] and partial response $[\mathrm{PR}]$ ) and DCR (proportion of patients with CR, $\mathrm{PR}$, and stable disease [SD]) were presented with Clopper-Pearson exact 95\% CIs. Progression-free survival defined as the time from the date of enrollment to Investigator-assessed disease progression confirmed radiologically, or death, was analyzed by Kaplan-Meier methodology; missing data for PFS were censored.

\section{Comparison of PK Parameters Between Chinese, Japanese, and US Patients}

Two Phase 1 studies investigated PK parameters of FTD/TPI in Japanese ${ }^{18}$ and $\mathrm{US}^{17}$ patients with solid tumors. As these studies were sponsored by Taiho Pharmaceutical Co Ltd and Taiho Oncology Inc, the data for subsets of patients treated with FTD/TPI $35 \mathrm{mg} / \mathrm{m}^{2}$ dose, as well as baseline characteristics of these patients, were available from clinical study reports and were used to compare PK parameters of FTD, TPI, and FTY between Chinese, Japanese, and US patients. Since PK parameters such as $\mathrm{C}_{\max }$ and AUC generally follow the lognormal distribution, and the usually limited sample size in a PK study precludes a reliable determination of the distribution of the data set, we have followed the FDA recommendation for the logarithmic transformation of PK data and calculation of geometric means for comparison purposes. ${ }^{32}$ Hence, in our study individual PK parameters that did not follow the Gaussian distribution (all except $\mathrm{T}_{\max }$ ) were converted into common logarithmic values, expressed as geometric means, and compared using the Tukey-Kramer's HSD multiple comparison test. The difference was considered significant at $p<0.05$. PK samples collected on days 1 and 12 were compared. The last PK sampling points were 10,10 , and $12 \mathrm{~h}$ for studies in China, Japan, and the US, respectively.

\section{Results}

\section{Demographic and Baseline}

\section{Characteristics}

Fifteen Chinese patients (12 male, 3 female) were enrolled in the study and received FTD/TPI; the majority of patients $(\mathrm{n}=13$ [86.7\%]) were diagnosed with CRC. The median (range) age was 56.0 (35-64) years, median (range) height, $170.0 \quad(155-183) \mathrm{cm}$, median (range) weight, 71.0 $(47-108) \mathrm{kg}$, and median body surface area, 1.86 (1.4-2.3) $\mathrm{m}^{2}$ (Table 1). All patients had undergone prior 
Table I Baseline Clinical and Demographic Characteristics (Safety Set)

\begin{tabular}{|l|l|}
\hline Characteristics & FTD/TPI (N= I5) \\
\hline Age, median (range), years & $56.0(35-64)$ \\
Male, $\mathrm{n}$ (\%) & $12(80.0)$ \\
Height, median (range), cm & $170.0(155.0-183.0)$ \\
Weight, median (range), $\mathrm{kg}$ & $71.0(47.0-108.0)$ \\
BMI, median (range), $\mathrm{kg} / \mathrm{m}^{2}$ & $25.6(19.6-32.3)$ \\
BSA, median (range), ${ }^{2}$ & $1.86(1.43-2.30)$ \\
\hline ECOG performance status, $\mathrm{n}(\%)$ & \\
0 & $4(26.7)$ \\
I & $11(73.3)$ \\
\hline No. of prior regimens, $\mathrm{n}(\%)$ & \\
2 & $5(33.3)$ \\
3 & $2(13.3)$ \\
$\geq 4$ & $8(53.3)$ \\
\hline Type of solid tumor, $\mathrm{n}(\%)$ & \\
Colorectal carcinoma & $13(86.7)$ \\
Breast cancer & $1(6.7)$ \\
Pancreatic carcinoma & $1(6.7)$ \\
\hline No. of nontarget lesions, $\mathrm{n}(\%)$ & \\
I & $3(20.0)$ \\
2 & $8(53.3)$ \\
3 & $2(13.3)$ \\
$\geq 4$ & $2(13.3)$ \\
\hline
\end{tabular}

Abbreviations: BMI, body mass index; BSA, body surface area; ECOG, Eastern Cooperative Oncology Group; FTD/TPI, trifluridine/tipiracil; SD, standard deviation.

anticancer therapy for advanced or metastatic disease, and $10(66.7 \%)$ patients had prior adjuvant anticancer therapy. Five (33.3\%) patients had received two prior regimens, two $(13.3 \%)$ had three prior regimens, and eight (53.3\%) had $\geq 4$ prior regimens. The primary tumor was resected in 12 $(80.0 \%)$ patients before enrollment; three $(20.0 \%)$ patients did not undergo resection. The median (range) time from the initial diagnosis to enrollment was 21.6 (8.0-215.2) months. Disease progression was reported as the outcome of prior therapy in all patients. The most frequent prior systemic cancer therapy was capecitabine monotherapy (in five $[33.3 \%]$ patients).

\section{Pharmacokinetic Characteristics}

Figure 1 shows mean plasma concentration-time profiles of FTD (A), FTY (B), and TPI (C) on days 1 and 12. Geometric means of PK parameters (all except $\mathrm{T}_{\max }$, which was reported as median) of FTD, FTY, and TPI analyzed on days 1 and 12 are presented in Table 2. FTD plasma concentrations on day 1 reached a $\mathrm{C}_{\max }$ of $3019.5 \mathrm{ng} / \mathrm{mL}$, at a median $\mathrm{T}_{\max }$ of $1 \mathrm{~h}$ after oral administration, and $\mathrm{AUC}_{0-\mathrm{t}}$ was $7796.6 \mathrm{ng} / \mathrm{mL} \cdot \mathrm{h}$. On day $12, \mathrm{C}_{\max }$ for FTD increased to $3693.1 \mathrm{ng} / \mathrm{mL}$, at a median $\mathrm{T}_{\max }$ of $2 \mathrm{~h}$ after administration, and $\mathrm{AUC}_{0-\mathrm{t}}$ was $18,181.3 \mathrm{ng} / \mathrm{mL} \cdot \mathrm{h}$; the $\mathrm{R}$ was 2.4 .

For FTY, $\mathrm{C}_{\max }$ value on day $1 \mathrm{was} 810.8 \mathrm{ng} / \mathrm{mL}$ at a median $\mathrm{T}_{\text {max }}$ of $1.5 \mathrm{~h}$ after administration, and $\mathrm{AUC}_{0-\mathrm{t}}$ was $3087.4 \mathrm{ng} /$ $\mathrm{mL} \cdot \mathrm{h}$. On day 12 , plasma concentrations of FTY reached a $\mathrm{C}_{\max }$ of $516.1 \mathrm{ng} / \mathrm{mL}$, at a median $\mathrm{T}_{\max }$ of $4 \mathrm{~h}$ after administration, and $\mathrm{AUC}_{0-\mathrm{t}}$ was $3512.1 \mathrm{ng} / \mathrm{mL} \bullet \mathrm{h}$; the $\mathrm{R}$ was 1.1 .

For TPI, $\mathrm{C}_{\max }$ was $60.0 \mathrm{ng} / \mathrm{mL}$ on day 1 , at a median $\mathrm{T}_{\max }$ of $2 \mathrm{~h}$, and $\mathrm{AUC}_{0-\mathrm{t}}$ was $247.3 \mathrm{ng} / \mathrm{mL} \cdot \mathrm{h}$. On day 12 , $\mathrm{C}_{\max }$ was $49.4 \mathrm{ng} / \mathrm{mL}$, at a median $\mathrm{T}_{\max }$ of $4 \mathrm{~h}$, and $\mathrm{AUC}_{0-\mathrm{t}}$ was $250.7 \mathrm{ng} / \mathrm{mL} \cdot \mathrm{h}$; the $\mathrm{R}$ was 1.0 (Table 2).

\section{Comparison of PK Parameters Between Chinese, Japanese, and US Patients}

This analysis included PK data from six Japanese patients (days 1 and 12), PK data on day 1 from the 19 US patients, and on day 12 from 34 US patients, and PK data on day 1
A

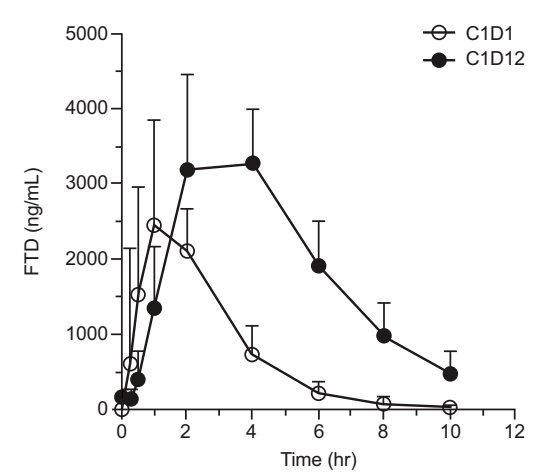

B

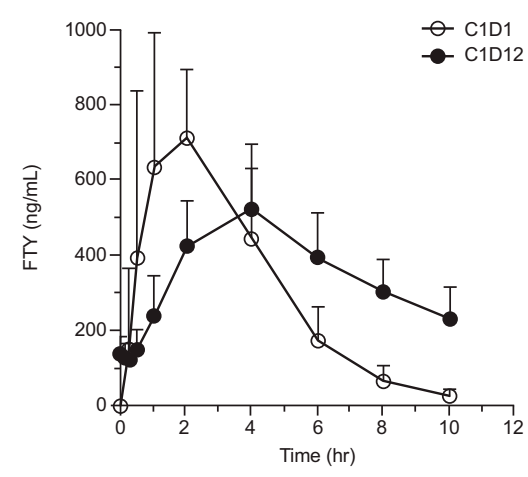

C

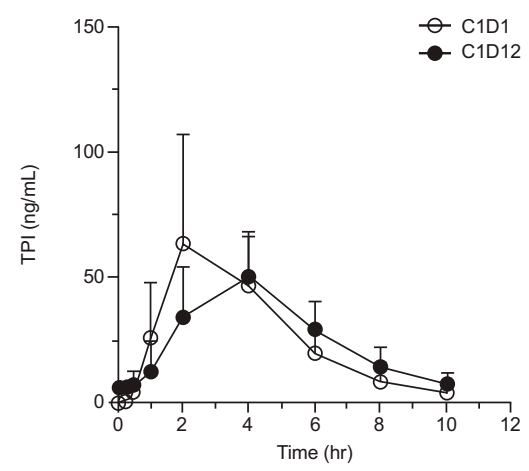

Figure I Mean plasma concentration-time profiles of FTD (A), FTY (B), and TPI (C) on days I and I2.

Abbreviations: CIDI, Cycle I day I; CIDI2, Cycle I day I2; FTD, trifluridine; FTY, inactive metabolite of FTD; hr, hour; TPI, tipiracil. 
Table 2 Geometric Means of PK Parameters for FTD, FTY, and TPI (PK Analysis Set)

\begin{tabular}{|c|c|c|c|c|c|c|}
\hline & \multicolumn{2}{|l|}{ FTD } & \multicolumn{2}{|l|}{ FTY } & \multicolumn{2}{|l|}{ TPI } \\
\hline & $\begin{array}{l}\text { Day I } \\
(n=14)\end{array}$ & $\begin{array}{l}\text { Day I2 } \\
(n=13)\end{array}$ & $\begin{array}{l}\text { Day I } \\
(n=14)\end{array}$ & $\begin{array}{l}\text { Day I2 } \\
(n=13)\end{array}$ & $\begin{array}{l}\text { Day I } \\
(n=\mid 4)\end{array}$ & $\begin{array}{l}\text { Day I2 } \\
(n=13)\end{array}$ \\
\hline $\mathrm{T}_{\max }$ median (range), $\mathrm{h}$ & $1.0(0.3-2.0)$ & $2.0(2.0-4.0)$ & $1.5(0.5-4.0)$ & $4.0(2.0-4.0)$ & $2.0(2.0-4.0)$ & $4.0(2.0-4.0)$ \\
\hline $\mathrm{T}_{\max }$ mean $(\mathrm{SD}), \mathrm{h}$ & I.I (0.5) & $2.8(1.0)$ & $1.9(1.3)$ & $3.5(0.9)$ & $2.5(0.8)$ & $3.5(0.9)$ \\
\hline $\mathrm{C}_{\max }(\mathrm{CV} \%), \mathrm{ng} / \mathrm{mL}$ & $3019.5(34.0)$ & $3693.1(21.7)$ & $810.8(27.8)$ & $516.1(28.0)$ & $60.0(54.9)$ & $49.4(34.5)$ \\
\hline$t_{1 / 2}(C V \%), h$ & I.3 (25.5) & $1.9(30.8)$ & $1.5(18.9)$ & $5.5(45.4)$ & $1.7(9.9)$ & $2.1(32.1)$ \\
\hline $\mathrm{AUC}_{0-\mathrm{t}}(\mathrm{CV} \%), \mathrm{ng} / \mathrm{mL} \cdot \mathrm{h}$ & $7796.6(19.1)$ & $18,181.3(30.0)$ & $3087.4(18.1)$ & $35 \mid 2.1(23.0)$ & $247.3(45.6)$ & $250.7(33.8)$ \\
\hline $\mathrm{AUC}_{0 \text {-inf }}(\mathrm{CV} \%), \mathrm{ng} / \mathrm{mL} \cdot \mathrm{h}$ & $7871 . I(19.6)$ & - & $3156.9(18.4)$ & - & $257.6(45.1)$ & - \\
\hline Vd/F (CV\%), L & $15.7(24.0)$ & $8.7(20.0)$ & - & - & $295.4(43.7)$ & $329.1(40.2)$ \\
\hline $\mathrm{CL} / \mathrm{F}$ (CV\%), L/h & $8.2(22.7)$ & $3.2(32.5)$ & - & - & II 7.8 (43.3) & $107.4(39.6)$ \\
\hline $\mathrm{R}^{\mathrm{a}}(\mathrm{CV} \%)$ & \multicolumn{2}{|c|}{$2.41^{\mathrm{b}}(17.9)$} & \multicolumn{2}{|c|}{$1.11^{\mathrm{b}}(27.5)$} & \multicolumn{2}{|c|}{$0.95^{\mathrm{b}}(66.9)$} \\
\hline
\end{tabular}

Notes: All PK parameters are presented as geometric means, except $\mathrm{T}_{\max }$, which is presented as median and mean. Geometric means are accompanied by the coefficient of variation (CV\%). ${ }^{a} \mathrm{R}=\left(\mathrm{AUC}_{0-\mathrm{t}}\right.$ [day I2]/AUC ${ }_{0-\mathrm{t}}\left[\right.$ day I]); ${ }^{\mathrm{b}} \mathrm{n}=12$; three patients had to be excluded from this analysis due to protocol violation.

Abbreviations: $A U_{0-\mathrm{t}}$, area under the plasma-concentration time curve from time 0 to time of last quantifiable concentration; $A U C_{0-i n f}$ area under the plasmaconcentration time curve (time 0 to time infinity); CL/F, apparent systemic clearance; $\mathrm{C}_{\text {max }}$, maximum plasma concentration; FTD, trifluridine; FTY, 5 -trifluoromethyluracil; PK, pharmacokinetic; SD, standard deviation; $t_{1 / 2}$, terminal half-life; $T_{\max }$, time to maximum concentration; TPI, tipiracil; Vd/F, apparent volume of distribution.

from 14 Chinese patients (one was excluded due to time violation regarding drug administration and PK sample collection), and on day 12 from 13 Chinese patients (two were excluded due to deviation in the time of sample collection). All patients included in this analysis were treated with oral FTD/TPI $35 \mathrm{mg} / \mathrm{m}^{2}$ twice daily. Baseline characteristics of the Japanese and US patients, included in the comparison, were: median (range) age 61.5 (53-68) years and 57.0 (37-74) years, median (range) weight $52.4(40-54) \mathrm{kg}$ and 83.9 (50-110) $\mathrm{kg}$, median (range) body surface area $1.4(1.3-1.6) \mathrm{m}^{2}$ and 1.94 (1.5-2.4) $\mathrm{m}^{2}$, respectively. Half of the patients were males, and the majority of patients in both studies were diagnosed with $\mathrm{CRC}$ and received $\geq 3$ prior treatments.

Geometric means for $\mathrm{C}_{\max }$ and $\mathrm{AUC}_{0-\mathrm{t}}$ from Chinese, Japanese, and US patients are presented in Table 3. TukeyKramer's HSD multiple comparison test of FTD $\mathrm{C}_{\max }$ and $\mathrm{AUC}_{0-\mathrm{t}}$ showed no significant differences on days 1 and 12

Table 3 Geometric Means of PK Parameters of Chinese, Japanese, and US Patients (PK Analysis Set ${ }^{\mathrm{a}}$ )

\begin{tabular}{|c|c|c|c|c|c|c|}
\hline \multirow[t]{2}{*}{ Region } & \multicolumn{3}{|c|}{ Day I } & \multicolumn{3}{|c|}{ Day 12} \\
\hline & $\mathbf{N}$ & $C_{\max }(\mathrm{ng} / \mathrm{mL})$ & $\begin{array}{l}\text { AUC }_{0-\mathrm{t}} \\
(\mathrm{ng} / \mathrm{mL} \cdot \mathrm{h})\end{array}$ & $\mathbf{N}$ & $C_{\max }(\mathrm{ng} / \mathrm{mL})$ & $\begin{array}{l}A^{A U C} C_{0-t} \\
(n g / m L \cdot h)\end{array}$ \\
\hline \multicolumn{7}{|l|}{ FTD } \\
\hline China & 14 & 3019.5 & 7796.6 & 13 & 3693.1 & $18,181.3$ \\
\hline Japan & 6 & 3254.6 & 8431.5 & 6 & 4457.6 & $20,852.1$ \\
\hline US & 19 & $2155.2^{\mathrm{a}}$ & $6618.1^{a}$ & 34 & 4474.3 & $22,563.0$ \\
\hline \multicolumn{7}{|l|}{ FTY } \\
\hline China & 14 & 810.8 & 3087.4 & 13 & 516.1 & 3512.1 \\
\hline Japan & 6 & 849.4 & 3334.4 & 6 & 553.9 & 3507.8 \\
\hline US & 19 & $736.8^{\mathrm{a}}$ & $3231.7^{\mathrm{a}}$ & $34^{\mathrm{b}}$ & 653.0 & 4875.6 \\
\hline \multicolumn{7}{|l|}{ TPI } \\
\hline China & 14 & 60.0 & 247.3 & 13 & 49.4 & 250.7 \\
\hline Japan & 6 & 69.7 & 271.0 & 6 & 59.5 & 278.3 \\
\hline US & 19 & 61.6 & 278.4 & 34 & 64.0 & 348.8 \\
\hline
\end{tabular}

Notes: Data from the Japanese and US patients were obtained from clinical study reports for a Phase I study in Japanese patients (JapicCTI-I I I545) and a Phase I study in

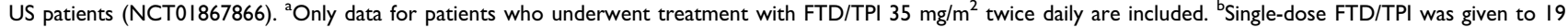
patients and single-dose FTD alone was given to 20 patients. All these patients subsequently received at least one cycle of FTD/TPI, with 34 patients available for PK analysis. Abbreviations: $\mathrm{AUC}_{0-\mathrm{t}}$, area under the plasma-concentration time curve from time 0 to time of last quantifiable concentration; $\mathrm{C}_{\text {max }}$, maximum plasma concentration; FTD, trifluridine; FTY, 5-trifluoromethyluracil; PK, pharmacokinetics; TPI, tipiracil. 
between Chinese and Japanese patients, Chinese and US patients, and Japanese and US patients (Figure 2). For FTY, $\mathrm{C}_{\max }$ and $\mathrm{AUC}_{0-\mathrm{t}}$ on day 12 were significantly higher $(p<0.05)$ in the US vs Chinese patients (Table 4). For TPI, there was no significant difference in $\mathrm{C}_{\max }$ on days 1 and 12 between the Chinese, Japanese, and US patients (Figure 3A and B), but $\mathrm{AUC}_{0-\mathrm{t}}$ on day 12 in US patients was significantly higher $(p<0.05)$ than in Chinese patients (Table 4, Figure 3D). There were no significant differences in $\mathrm{AUC}_{0-\mathrm{t}}$ for TPI on day 12 between Chinese and Japanese patients, or between Japanese and US patients (Table 4, Figure 3D).

\section{Treatment Exposure and Safety}

Most Chinese patients received FTD/TPI treatment for one or two cycles: all 15 received one cycle of FTD/TPI, 13 $(86.7 \%)$ received two cycles, one $(6.7 \%)$ received three cycles, and one $(6.7 \%)$ received $\geq 4$ cycles. The median (range) FTD/TPI treatment exposure was 8.1 (4.0-18.9)

B

A

FTD, C1D1
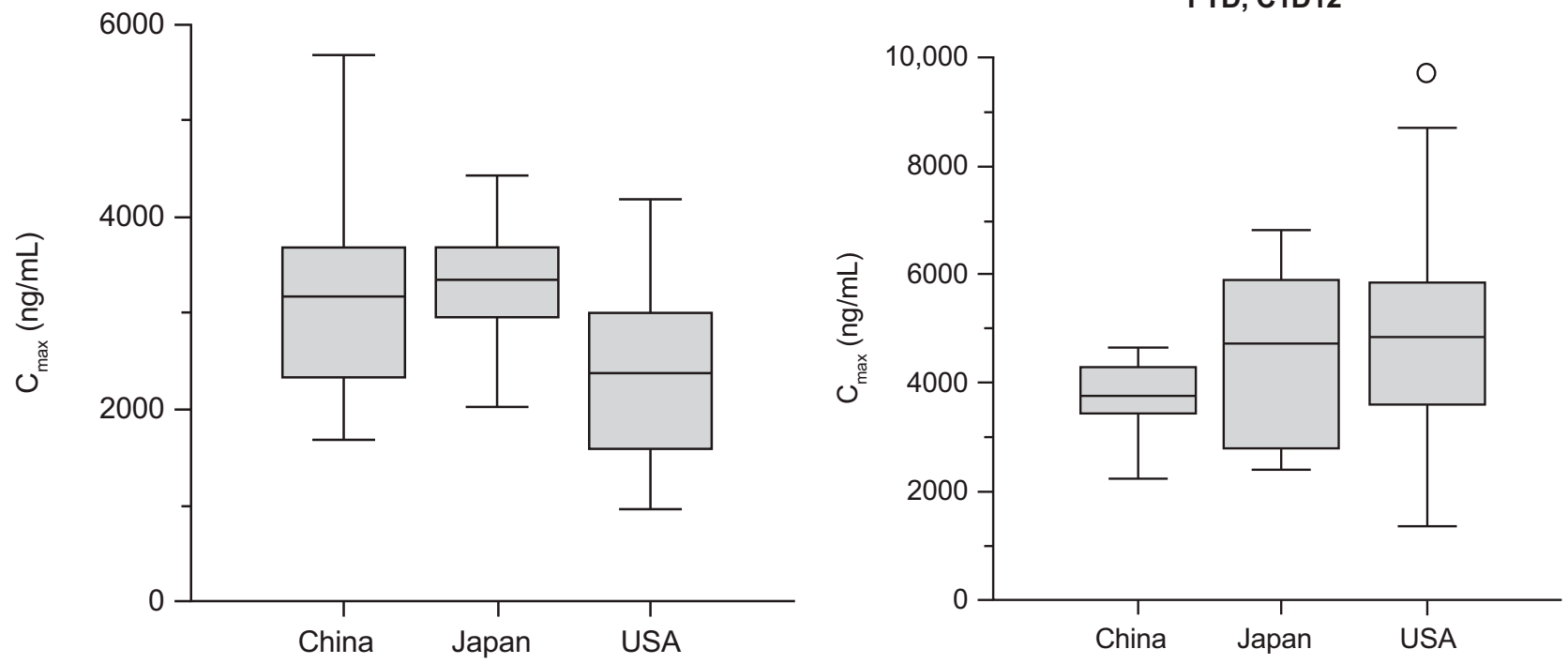

C

FTD, C1D1

D

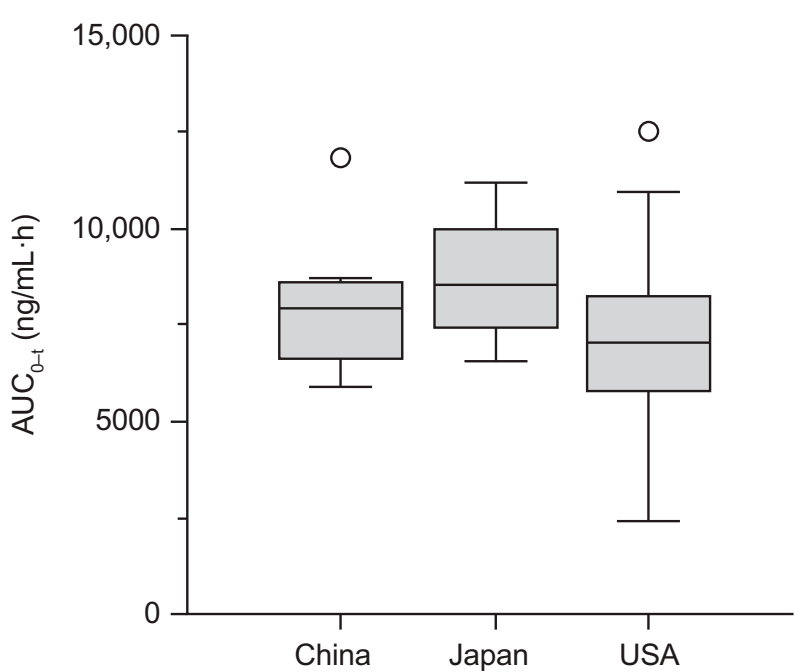

FTD, C1D12

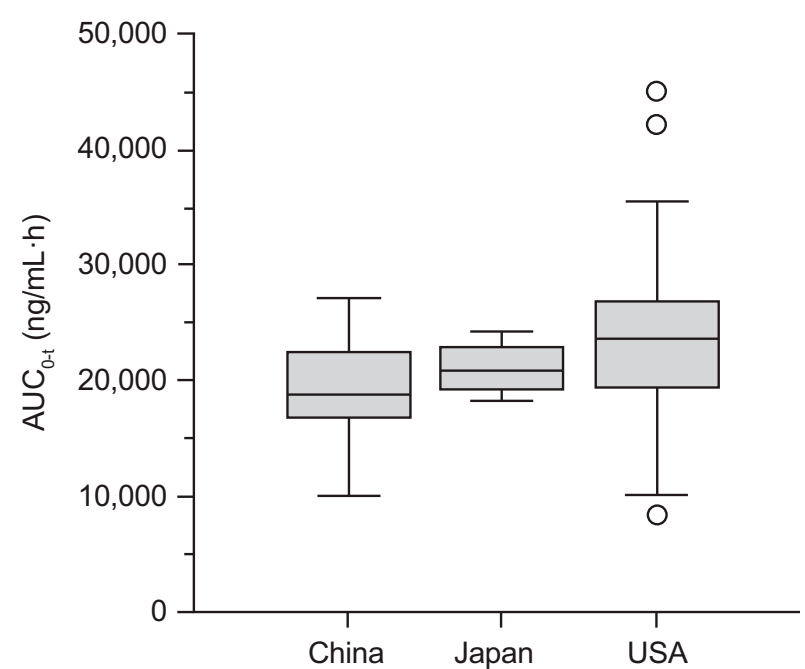

Figure 2 Box plots of $C_{\max }(\mathbf{A}, \mathbf{B})$ and $A \cup C_{0-t}(\mathbf{C}, \mathbf{D})$ of FTD after administration of FTD/TPI on day I (CIDI) and day I2 (CIDI2) of Cycle I by regions of China, Japan, and the US. Differences between regions were assessed by Tukey-Kramer's HSD multiple comparison test. Data for Japan and the US used for the comparison are presented in Table 3. Upper horizontal line of box indicates 75th percentile; lower horizontal line of box, 25th percentile; horizontal bar within box, median; upper horizontal bar outside box, 90th percentile; lower horizontal bar outside box, 10th percentile; circles represent outliers.

Abbreviations: $\mathrm{AUC}_{0-\mathrm{t}}$, area under the plasma-concentration time curve from time 0 to time of last quantifiable concentration; $\mathrm{C}$, cycle; $\mathrm{C}_{\max }$, maximum plasma concentration; D, day; FTD, trifluridine; HSD, honestly significant difference; TPI, tipiracil. 


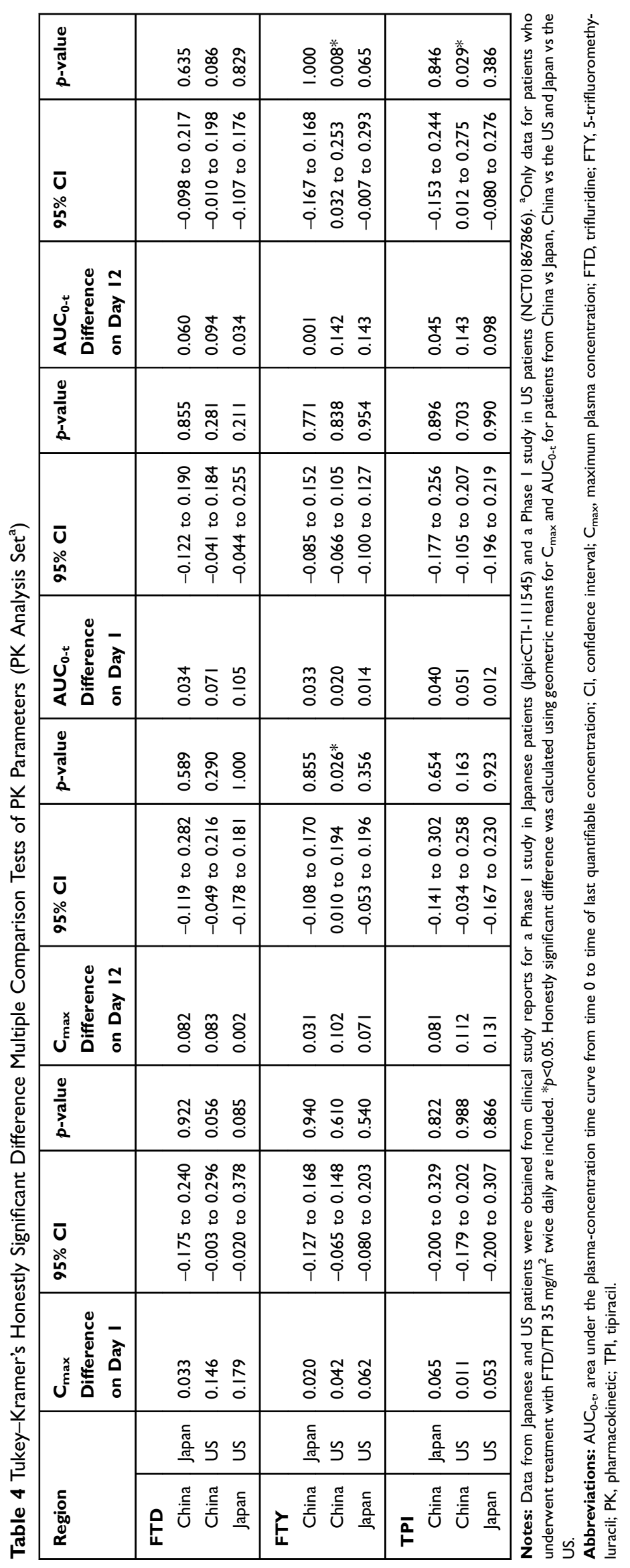


A

TPI, C1D1

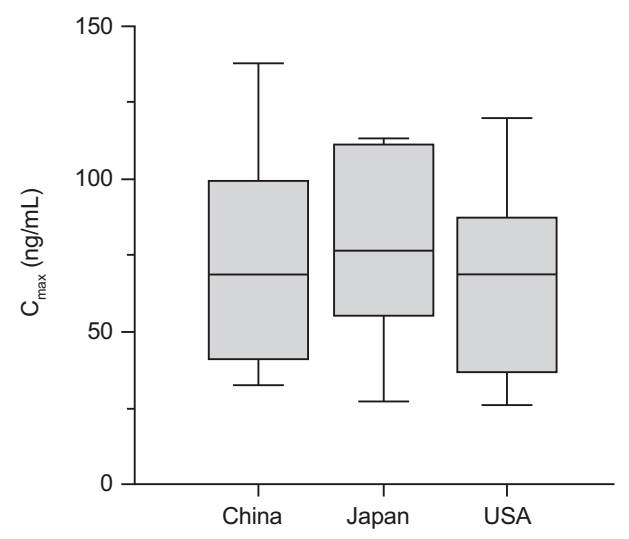

C

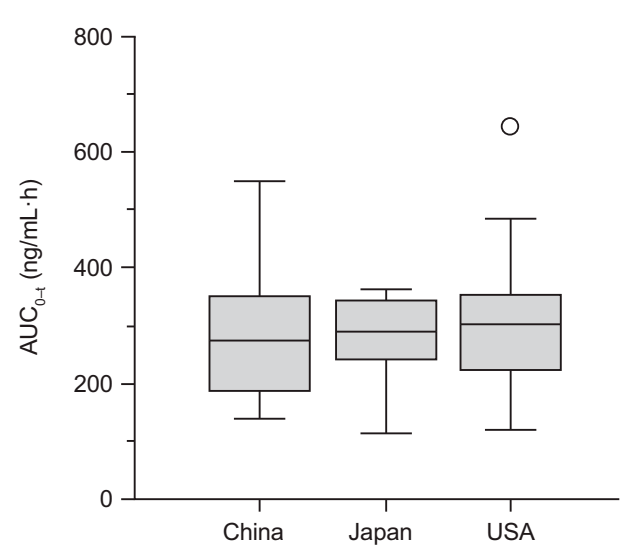

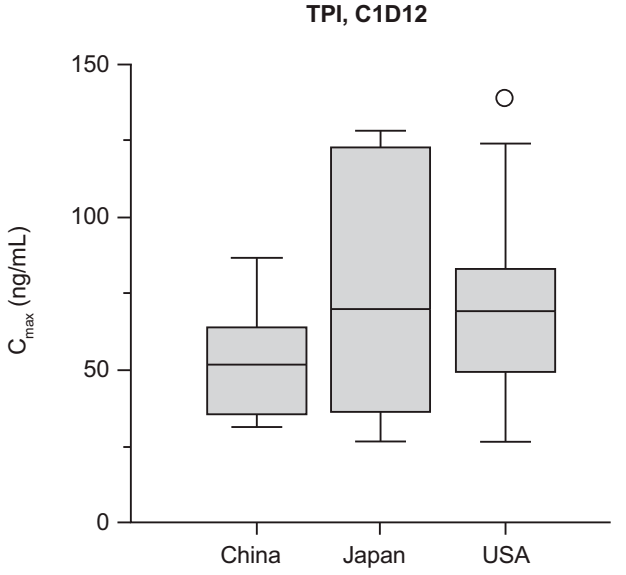

D

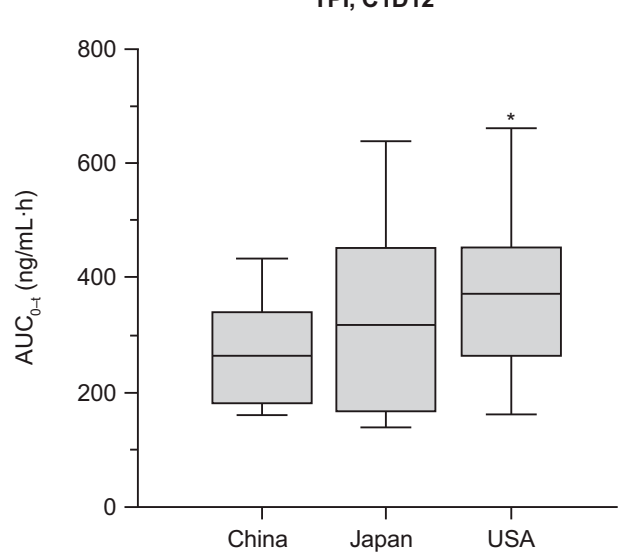

Figure 3 Box plots of $\mathrm{C}_{\max }(\mathbf{A}, \mathbf{B})$ and $\mathrm{AUC}_{0-\mathrm{t}}(\mathbf{C}, \mathbf{D})$ of TPI after administration of FTD/TPI on day I (CIDI) and day I2 (CIDI2) of cycle I by regions of China, Japan, and the US. Data for Japan and the US used for the comparison are presented in Table 3. Differences between regions were assessed by Tukey-Kramer's honestly significant difference multiple comparison test. *p<0.05 compared with China. Upper horizontal line of box indicates 75 th percentile; lower horizontal line of box, 25 th percentile; horizontal bar within box, median; upper horizontal bar outside box, 90th percentile; lower horizontal bar outside box, I0th percentile; circles represent outliers.

Abbreviations: $\mathrm{AUC}_{0-\mathrm{t}}$, area under the plasma-concentration time curve from time 0 to time of last quantifiable concentration; $\mathrm{C}$, cycle; $\mathrm{C}_{\text {max }}$ maximum plasma concentration; D, day; FTD, trifluridine; TPI, tipiracil; US, United States.

weeks. Per the protocol-defined discontinuation criteria, RECIST-defined disease progression was the reason for treatment discontinuation for $14(93.3 \%)$ patients; one patient $(6.7 \%)$ discontinued at their own request.

Overall, five patients had a dose delay/interruption due to AEs (anemia, constipation, platelet count decreased, and leukopenia in one patient each; neutrophil count decreased and neutropenia in two patients each). In addition, one (6.7\%) patient had a dose reduction after Cycle 1 because of decreased white blood cell and neutrophil counts.

The mean (standard deviation) overall study medication compliance rate was $93.7 \%$ (12.3), with compliance rates of $99.3 \%, 88.9 \%, 100 \%$, and $50 \%$ for Cycles $1-4$, respectively. Twelve $(80 \%)$ patients were in the $90 \%$ to $\leq 100 \%$ compliance range; one $(6.7 \%)$ patient was in the $80 \%-\leq 90 \%$ compliance range.

All 15 patients were included in the safety analyses. Most patients $(n=14$ [93.3\%]) experienced $\geq 1$ AE, and 13 $(86.7 \%)$ experienced $\geq 1$ treatment-emergent AE (TEAE). One $(6.7 \%)$ patient experienced TEAEs of nausea, vomiting, dizziness, and headache that led to discontinuation from study treatment; however, none of these were related to study treatment. Eight patients (53.3\%) experienced Grade 3 TEAEs, of which the most frequent were anemia and fatigue (two [13.3\%] patients for each event); no Grade 4 TEAEs were observed (Table 5).

The most frequent hematologic TEAEs included neutropenia $(n=5[33.3 \%])$, leukopenia $(n=5[33.3 \%])$, anemia $(n=4$ [26.7\%]), neutrophil count decreased $(n=4[26.7 \%])$, and 
Table 5 TEAEs in $\geq 10 \%$ of Patients (Safety Set) $(n=15)$

\begin{tabular}{|c|c|c|}
\hline TEAEs & $\begin{array}{l}\text { All } \\
\text { Grades } \\
\text { n (\%) }\end{array}$ & $\begin{array}{l}\text { Grade } \\
3 \\
\text { n (\%) }\end{array}$ \\
\hline Patients with $\geq I$ TEAE & $13(86.7)$ & $8(53.3)$ \\
\hline $\begin{array}{l}\text { Blood and lymphatic system disorders } \\
\text { Leukopenia } \\
\text { Neutropenia } \\
\text { Anemia } \\
\text { Erythropenia }\end{array}$ & $\begin{array}{l}5(33.3) \\
5(33.3) \\
4(26.7) \\
2(13.3)\end{array}$ & $\begin{array}{l}0 \\
1(6.7) \\
2(13.3) \\
0\end{array}$ \\
\hline $\begin{array}{l}\text { Investigations } \\
\text { Neutrophil count decreased } \\
\text { White blood cell count decreased } \\
\text { Aspartate aminotransferase increased } \\
\text { Hemoglobin decreased } \\
\text { Platelet count decreased }\end{array}$ & $\begin{array}{l}4(26.7) \\
4(26.7) \\
2(13.3) \\
2(13.3) \\
2(13.3)\end{array}$ & $\begin{array}{l}I(6.7) \\
I(6.7) \\
I(6.7) \\
0 \\
I(6.7)\end{array}$ \\
\hline $\begin{array}{l}\text { Gastrointestinal disorders } \\
\text { Nausea } \\
\text { Vomiting } \\
\text { Constipation } \\
\text { Diarrhea } \\
\text { Abdominal distension } \\
\text { Abdominal pain } \\
\text { Abdominal pain, upper }\end{array}$ & $\begin{array}{l}12(80.0) \\
9(60.0) \\
5(33.3) \\
3(20.0) \\
2(13.3) \\
2(13.3) \\
2(13.3)\end{array}$ & $\begin{array}{l}I(6.7) \\
I(6.7) \\
0 \\
0 \\
0 \\
0 \\
0\end{array}$ \\
\hline $\begin{array}{l}\text { General disorders and administration site } \\
\text { conditions } \\
\text { Fatigue }\end{array}$ & $8(53.3)$ & $2(13.3)$ \\
\hline $\begin{array}{l}\text { Metabolism and nutrition disorders } \\
\text { Decreased appetite } \\
\text { Hyperglycemia }\end{array}$ & $\begin{array}{l}7(46.7) \\
3(20.0)\end{array}$ & $\begin{array}{l}0 \\
0\end{array}$ \\
\hline $\begin{array}{l}\text { Nervous system disorders } \\
\text { Dizziness } \\
\text { Headache }\end{array}$ & $\begin{array}{l}3(20.0) \\
2(13.3)\end{array}$ & $\begin{array}{l}\text { I (6.7) } \\
\text { I (6.7) }\end{array}$ \\
\hline $\begin{array}{l}\text { Musculoskeletal and connective tissue } \\
\text { disorders } \\
\text { Back pain }\end{array}$ & $2(13.3)$ & 0 \\
\hline $\begin{array}{l}\text { Infections and infestations } \\
\text { Upper RTI }\end{array}$ & $2(13.3)$ & 0 \\
\hline $\begin{array}{l}\text { Ear and labyrinth disorders } \\
\text { Tinnitus }\end{array}$ & $2(13.3)$ & 0 \\
\hline
\end{tabular}

Abbreviations: RTI, respiratory tract infection; TEAE, treatment-emergent adverse event.

white blood cell count decreased ( $n=4$ [26.7\%]). Nonhematologic toxicity consisted mainly of nausea $(n=12$ $[80.0 \%])$, vomiting $(n=9[60.0 \%])$, fatigue $(n=8[53.3 \%])$, decreased appetite $(n=7 \quad[46.7 \%])$, constipation $(n=5$ $[33.3 \%])$, diarrhea $(n=3[20.0 \%])$, hyperglycemia $(n=3$
[20.0\%]), and dizziness ( $\mathrm{n}=3$ [20.0\%]) (Table 5). Four (26.7\%) patients experienced serious TEAEs; three of these (anemia, fatigue, and decreased platelet count) were considered related to study treatment. No TEAE-related deaths were reported in this study.

\section{Efficacy}

Preliminary efficacy data were available for all 15 Chinese patients. The ORR was 0 and DCR was $13.3 \%$ (95\% CI, 1.7-40.5). The best response was stable disease in two (13.3\%) patients and progressive disease in $13(86.7 \%)$ patients. Median PFS was 1.9 months (95\% CI, 1.3-2.1). One $(6.7 \%)$ patient was censored because of treatment discontinuation. At 3 months, per Kaplan-Meier estimate, $8.3 \%(95 \% \mathrm{CI}, 0.5-30.6)$ of patients were alive and $6.7 \%$ (95\% CI, 0.2-31.9) were at risk (Figure 4).

\section{Discussion}

This phase $1 \mathrm{~b}$ study investigated the $\mathrm{PK}$, safety, and preliminary efficacy of oral FTD/TPI in Chinese patients with solid tumors, mostly CRC. The exposure to FTD increased 2.4-fold with multiple dosing, and a similar tendency was previously observed in similar Phase 1 studies in Japanese ${ }^{18}$ and US patients. ${ }^{17}$ Comparison of the PK parameters of FTD/TPI obtained in this study with original clinical study results from Japanese (JapicCTI-111545) and US (NCT01867866) patients, based on Tukey-Kramer's HSD multiple comparison test, revealed that the exposures of FTD, which is an active compound of FTD/TPI, were similar between Chinese, Japanese, and US patients on days 1 and 12. Although the exposure to TPI on day 12 in Chinese patients was lower than that in US patients, it was comparable between Chinese and Japanese patients. A limitation of this analysis was a small sample size, particularly for Japanese patients $(\mathrm{N}=6)$.

FTD/TPI was safe and tolerable in this study, and the safety data observed in Chinese patients were similar to those reported in other studies of FTD/TPI in solid tumors or mCRC, with the main toxicities affecting the hematologic and gastrointestinal systems. ${ }^{15,17-19,21,22,25}$ Although the safety profile generally complemented results reported in the global RECOURSE ${ }^{22}$ and Asian TERRA trials, ${ }^{25}$ the frequency of most common hematologic and gastrointestinal AEs, including Grade 3 AEs, was lower in this study, likely due to small sample size and relatively short treatment exposure.

No CR or PR was observed in this study and the best response was stable disease, similar to the results of a Phase 1 


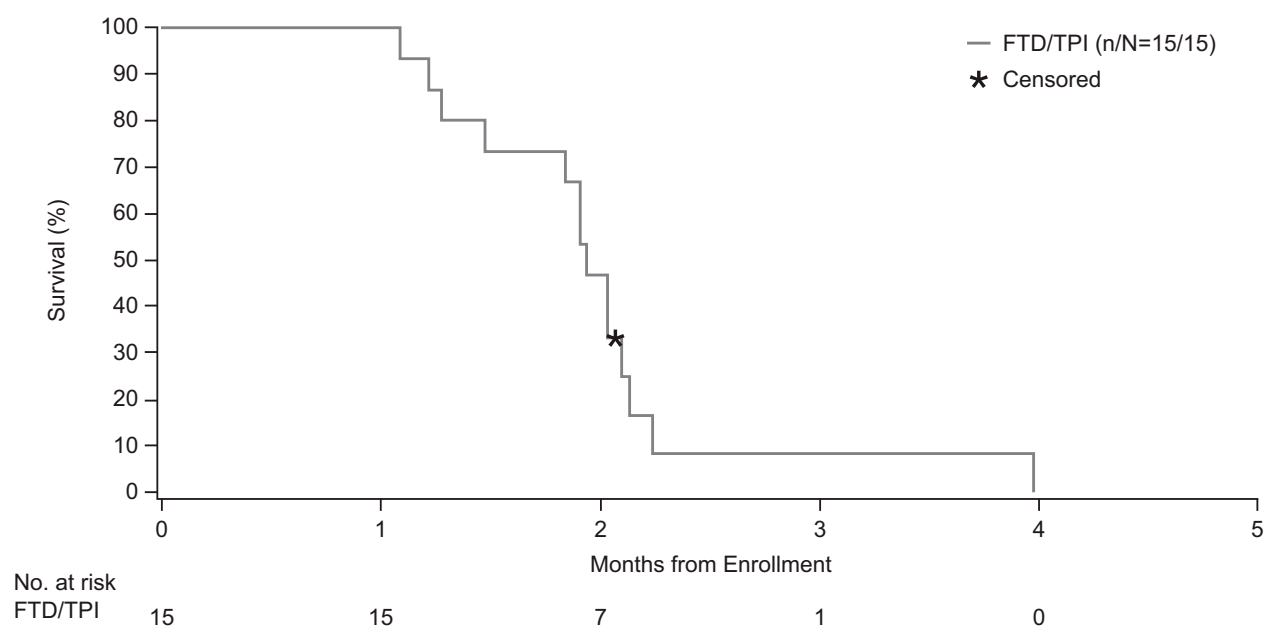

Figure 4 Progression-free survival (efficacy set). Estimation by Kaplan-Meier method. Abbreviations: FTD, trifluridine; TPI, tipiracil.

Japanese study. ${ }^{18}$ Global RECOURSE ${ }^{22}$ and Asian TERRA trials $^{25}$ reported ORRs of $1.6 \%$ and $1.1 \%$, respectively. Median PFS of 1.9 months in this study was comparable, with the caveat of small sample size, to that in the Japanese PK study (2.6 months), ${ }^{18}$ as well as to the RECOURSE ${ }^{22}$ and TERRA trials ${ }^{25}$ ( 2 months in both). Indeed, the major limitation of this study in relation to efficacy outcomes was that the sample size was too small to draw any definitive conclusions. Nevertheless, the study met its primary objective to obtain preliminary information on PK and toxicity profiles of FTD/ TPI in Chinese patients.

\section{Conclusions}

In summary, the PK profile of FTD/TPI in this study was comparable between Chinese, Japanese, and US patients. The treatment was safe and tolerable, and the safety profile was similar to that reported in large randomized trials, ${ }^{22,25}$ with no new safety signals identified. The PK characteristics, as well as the safety and preliminary efficacy results of FTD/TPI reported herein, taken in context of the results reported by large the RECOURSE and TERRA trials, ${ }^{22,25}$ suggest that FTD/TPI could provide a new treatment option for Chinese patients with $\mathrm{mCRC}$ refractory to standard therapies. As FTD/TPI has an antitumor effect irrespective of KRAS mutations, ${ }^{22}$ a broad mCRC patient population may derive clinical benefit from this salvage therapy.

\section{Data-Sharing Statement}

Data collected for this study, including individual patient data, will not be made available.

\section{Acknowledgments}

The authors thank Dr Hupei, from the Phase 1 clinical pharmacology center of Peking Union Medical College Hospital, for assistance in PK sample processing and storage, and Kunihiro Yoshisue, PhD, Pharmacokinetics Research Lab, Taiho Pharmaceutical Co., Ltd, for analyzing PK data. Medical writing assistance was supported by Taiho Pharmaceutical Co Ltd, and was provided by Anoop Joseph, MSc, and Ewa Wandzioch, $\mathrm{PhD}$, of Parexel International.

\section{Author Contributions}

Xicheng Wang, Chunmei Bai, Jianfeng Zhou, and Lin Shen contributed to the conception or design of the study; Xicheng Wang, Chunmei Bai, Jianfeng Zhou, Yan Li, Yuping Ge, Yanping Zhou, and Lin Shen participated in patient recruitment and follow-up, as well as generation and acquisition of clinical data. All authors contributed to data analysis, drafting and revising the article, provided final approval of the version to be published, and agreed to be accountable for all aspects of the work.

\section{Funding}

This work was supported by Taiho Pharmaceutical Co Ltd.

\section{Disclosure}

Xicheng Wang, Chunmei Bai, Jianfeng Zhou, Yan Li, Yuping Ge, and Yanping Zhou have no conflicts of interest to declare. Lin Shen acknowledges honoraria and speakers' fees from Novartis, Pfizer, Roche, Sanofi, and Taiho; has served as a consultant for Novartis, Roche, and Taiho; and acknowledges research funding from Hengrui 
Medicine, Roche, and Taiho. The authors report no other conflicts of interest in this work.

\section{References}

1. Bray F, Ferlay J, Soerjomataram I, Siegel RL, Torre LA, Jemal A. Global cancer statistics 2018: GLOBOCAN estimates of incidence and mortality worldwide for 36 cancers in 185 countries. CA Cancer J Clin. 2018;68(6):394-424. doi:10.3322/caac.v68.6

2. Van Cutsem E, Cervantes A, Adam R, et al. ESMO consensus guidelines for the management of patients with metastatic colorectal cancer. Ann Oncol. 2016;27(8):1386-1422. doi:10.1093/annonc/mdw235

3. Yoshino T, Arnold D, Taniguchi H, et al. Pan-Asian adapted ESMO consensus guidelines for the management of patients with metastatic colorectal cancer: a JSMO-ESMO initiative endorsed by CSCO, KACO, MOS, SSO and TOS. Ann Oncol. 2018;29(1):44-70. doi:10.1093/annonc/mdx 738

4. National Comprehensive Cancer Network (NCCN). NCCN Clinical Practice Guidelines in Oncology (NCCN Guidelines ${ }^{\circledR}$ ). Colon Cancer. Version 2. 2019 May 15.

5. National Comprehensive Cancer Network (NCCN). NCCN Clinical Practice Guidelines in Oncology (NCCN Guidelines $\left.{ }^{\circledR}\right)$. Rectal Cancer. Version 2. 2019 May 15.

6. Lenz HJ, Stintzing S, Loupakis F. TAS-102, a novel antitumor agent: a review of the mechanism of action. Cancer Treat Rev. 2015;41 (9):777-783. doi:10.1016/j.ctrv.2015.06.001

7. Emura T, Murakami Y, Nakagawa F, Fukushima M, Kitazato K. A novel antimetabolite, TAS-102 retains its effect on FU-related resistant cancer cells. Int J Mol Med. 2004;13(4):545-549.

8. Tanaka N, Sakamoto K, Okabe H, et al. Repeated oral dosing of TAS-102 confers high trifluridine incorporation into DNA and sustained antitumor activity in mouse models. Oncol Rep. 2014;32 (6):2319-2326. doi:10.3892/or.2014.3487

9. Fujiwara Y, Oki T, Heidelberger C. Fluorinated pyrimidines. XXXVII. Effects of 5-trifluoromethyl-2'-deoxyuridine on the synthesis of deoxyribonucleic acid of mammalian cells in culture. Mol Pharmacol. 1970;6(3):273-280.

10. Matsuoka K, Iimori $\mathrm{M}$, Niimi $\mathrm{S}$, et al. Trifluridine induces p53-dependent sustained G2 phase arrest with its massive misincorporation into DNA and few DNA strand breaks. Mol Cancer Ther. 2015;14(4):1004-1013. doi:10.1158/1535-7163.MCT-14-0236

11. Dexter DL, Wolberg WH, Ansfield FJ, Helson L, Heidelberger C. The clinical pharmacology of 5-trifluoromethyl-2'-deoxyuridine. Cancer Res. 1972;32(2):247-253.

12. Fukushima M, Suzuki N, Emura T, et al. Structure and activity of specific inhibitors of thymidine phosphorylase to potentiate the function of antitumor 2'-deoxyribonucleosides. Biochem Pharmacol. 2000;59(10):1227-1236. doi:10.1016/S0006-2952(00)00253-7

13. Emura T, Suzuki N, Fujioka A, Ohshimo H, Fukushima M. Potentiation of the antitumor activity of alpha, alpha, alpha-trifluorothymidine by the co-administration of an inhibitor of thymidine phosphorylase at a suitable molar ratio in vivo. Int J Oncol. 2005;27(2):449-455.

14. Hong DS, Abbruzzese JL, Bogaard K, et al. Phase I study to determine the safety and pharmacokinetics of oral administration of TAS-102 in patients with solid tumors. Cancer. 2006;107(6):13 83-1390. doi:10.1002/cncr.22125

15. Overman MJ, Kopetz S, Varadhachary G, et al. Phase I clinical study of three times a day oral administration of TAS-102 in patients with solid tumors. Cancer Invest. 2008;26(8):794-799. doi:10.1080/ 07357900802087242
16. Overman MJ, Varadhachary G, Kopetz S, et al. Phase 1 study of TAS-102 administered once daily on a 5-day-per-week schedule in patients with solid tumors. Invest New Drugs. 2008;26(5):445-454. doi:10.1007/s10637-008-9142-3

17. Cleary JM, Rosen LS, Yoshida K, Rasco D, Shapiro GI, Sun W. A phase 1 study of the pharmacokinetics of nucleoside analog trifluridine and thymidine phosphorylase inhibitor tipiracil (components of TAS-102) vs trifluridine alone. Invest New Drugs. 2017;35 (2):189-197. doi:10.1007/s10637-016-0409-9

18. Doi T, Ohtsu A, Yoshino T, et al. Phase I study of TAS-102 treatment in Japanese patients with advanced solid tumours. Br J Cancer. 2012;107(3):429-434. doi:10.1038/bjc.2012.274

19. Bendell JC, Rosen LS, Mayer RJ, et al. Phase 1 study of oral TAS-102 in patients with refractory metastatic colorectal cancer. Cancer Chemother Pharmacol. 2015;76(5):925-932. doi:10.1007/ s00280-015-2850-4

20. Yoshino T, Kojima T, Bando H, et al. Effect of food on the pharmacokinetics of TAS-102 and its efficacy and safety in patients with advanced solid tumors. Cancer Sci. 2016;107(5):659-665. doi:10.1111/cas.12912

21. Yoshino T, Mizunuma N, Yamazaki K, et al. TAS-102 monotherapy for pretreated metastatic colorectal cancer: a double-blind, randomised, placebo-controlled phase 2 trial. Lancet Oncol. 2012;13 (10):993-1001. doi:10.1016/S1470-2045(12)70345-5

22. Mayer RJ, Van Cutsem E, Falcone A, et al. Randomized trial of TAS-102 for refractory metastatic colorectal cancer. $N$ Engl J Med. 2015;372(20):1909-1919. doi:10.1056/NEJMoa1414325

23. Longo-Munoz F, Argiles G, Tabernero J, et al. Efficacy of trifluridine and tipiracil (TAS-102) versus placebo, with supportive care, in a randomized, controlled trial of patients with metastatic colorectal cancer from Spain: results of a subgroup analysis of the Phase 3 RECOURSE trial. Clin Transl Oncol. 2017;19(2):227-235. doi:10. 1007/s12094-016-1528-7

24. Kim TW, Shen L, Xu JM, et al. TERRA: a randomized, double-blind, placebo-controlled phase 3 study of TAS-102 in Asian patients with metastatic colorectal cancer. Ann Oncol. 2016;27(supp16):465PD. doi:10.1093/annonc/mdw370.14

25. Xu J, Kim TW, Shen L, et al. Results of a randomized, double-blind, placebo-controlled, Phase III trial of trifluridine/tipiracil (TAS-102) monotherapy in Asian patients with previously treated metastatic colorectal cancer: the TERRA study. J Clin Oncol. 2018;36 (4):350-358. doi:10.1200/JCO.2017.74.3245

26. Eisenhauer EA, Therasse P, Bogaerts J, et al. New response evaluation criteria in solid tumours: revised RECIST guideline (version 1.1). Eur J Cancer. 2009;45(2):228-247. doi:10.1016/j.ejca.2008.10.026

27. Common Terminology Criteria for Adverse Events (CTCAE) Version 4.0. (v4.03: June 14, 2010). Available from: https://evs.nci.nih.gov/ ftp 1/CTCAE/CTCAE_4.03/CTCAE_4.03_2010-06-14_ QuickReference_8.5x11.pdf. Accessed December 2, 2019.

28. Association WM. World Medical Association Declaration of Helsinki: ethical principles for medical research involving human subjects. JAMA. 2013;310(20):2191-2194. doi:10.1001/jama.2013.281053

29. ICH Expert Working Group. ICH harmonised tripartite guideline. Guideline for good clinical practice E6. Available from: http://www. ich.org/fileadmin/Public_Web_Site/ICH_Products/Guidelines/ Efficacy/E6/E6_R1_Guideline.pdf. Accessed July 12, 2019.

30. Center for Drug Evaluation (CFDA). Chemical drug clinical pharmacokinetics study technical guidelines. Available from: http://www.cde.org. cn/zdyz.do?method=largePage\&id=2070. Accessed August 5, 2019.

31. China Food and Drug Administration. Available from: http://www. sda.gov.cn/WS01/CL0053/24529.html. Accessed August 2, 2019.

32. Guidance for Industry Statistical Approaches to Establishing Bioequivalence. Available from: https://www.fda.gov/media/70958/ download. Accessed December 2, 2019. 


\section{Publish your work in this journal}

Clinical Pharmacology: Advances and Applications is an international, peer-reviewed, open access journal publishing original research, reports, reviews and commentaries on all areas of drug experience in humans. The manuscript management system is completely online and

includes a very quick and fair peer-review system, which is all easy to use. Visit http://www.dovepress.com/testimonials.php to read real quotes from published authors. 